\title{
Maximizing the Probability of Message Delivery over Ever-changing Communication Scenarios in Tactical Networks
}

\author{
Johannes F. Loevenich, Roberto Rigolin F. Lopes, Paulo H. L. Rettore, Sharath M. Eswarappa and Peter Sevenich
}

\begin{abstract}
This letter introduces a stochastic model to maximize the probability of message delivery over ever-changing communication scenarios in tactical networks. Our model improves modern tactical systems implementing store-and-forward mechanisms organized in a hierarchy of layers for messages, IP packets and radios. The goal is to compute close to optimal parameters for a transport protocol by computing the optimum redundancy for the user data-flow to overcome packet loss during changes in the link data rate, including disconnections. Experiments in a VHF network illustrate the numerical results from our model using messages with different sizes over two patterns of data rate change.
\end{abstract}

Index Terms-Tactical Networks, Ever-changing Communication Scenarios, Message Delivery, System Robustness.

\section{INTRODUCTION}

C OMMUNICATION scenarios at the edge of tactical networks are exposed to several sources of randomness that can change the radio link data rate [1]. Therefore, tactical systems might have to deliver messages over ever-changing scenarios with both user data-flows and network conditions changing independently [2]. Given the wide range of military operations, it is challenging to design tactical systems that can thrive in arbitrary communication scenarios, also including link disconnections (worst case). Multi-layer control mechanisms, within tactical systems, rely on feedback from the radio and router to compute the current link quality (e.g. data rate, latency, jitter and packet loss). The goal is to mitigate packet loss, doing flow control and adding redundancy, to increase the probability of message delivery over unreliable radio links.

We start with the hypothesis that the probability of message delivery can be computed and finally maximized using crosslayer information within a modern tactical system with interfaces to the radio, router, message queue and proxy/gateway. Our model assumes that the network conditions are quantized by a set of data rates updated within a time window. Thus, the probability of packet delivery is directly related to the amount of bits successfully transmitted during the time window. Assuming that the tactical system uses error correction techniques in different layers (e.g. [3]), the probability of

J. F. Loevenich, R. R. F. Lopes, P. H. Rettore, S. M. Eswarappa and P. Sevenich were with the Communication Systems Department (KOM), Fraunhofer FKIE, Bonn, 53177 Germany, e-mail: \{johannes.loevenich, roberto.lopes, paulo.lopes.rettore, peter.sevenich\}@fkie.fraunhofer.de.

J. F. Loevenich and S. M. Eswarappa were also with the Institute of Computer Science 4, University of Bonn, Bonn, Germany e-mail: \{s6joloev, s6shmali\}@uni-bonn.de

Manuscript received December 00, 2020; revised December 00, 2020. message delivery can be described by a binomial distribution, where $n$ is the number of packets including redundancy and $p$ is the probability that a single packet is delivered. As a result, our model goes layer-by-layer computing probabilities to estimate the probability of message delivery.

Our hypothesis is verified using two exemplary patterns of link data rate change discussed in [1]. There, we quantified the inter-packet latency of three types of data-flow over a VHF radio link changing in a given pattern. Here, we introduce a model to compute the probability of message delivery during those experiments. The goal is to calculate the close to optimal redundancy to increase the probability of message delivery over ever-changing network conditions including link disconnections.

\section{The Problem}

\section{A. Ever-changing communication scenario}

Modern tactical systems are organized into layers complementing each other through multi-layer control mechanisms to handle independent changes from both user data-flows $(A)$ and network conditions $(B)$, as illustrated in Fig. 1. This figure shows the end-to-end communication scenario with the sender and the receiver connected through a radio link, composing of an ever-changing communication scenario [1], [2]. Each node has a control plane $(c)$ and two chains: one for incoming (i) data-flows and another for outgoing (o) data-flows, both sitting in at least four layers, namely radio (layer 0), packet (layer 1), message (layer 2) and proxy/broker (layer 3). Notice the connection symmetry through a noisy channel, where the out chain from the sender is connected to the in chain of the receiver and vice-versa.

The sequence of messages from command and control systems (A) enter the system from layer 3 carrying a set of QoS requirements such as priority, reliability and time of expire (differentiated at layer 2), which are partially mapped to IP packets at layer 1 . And the radio (layer 0 ) usually has a buffer with limited size that differentiates the packets by priority. Note that a multi-homed node with $r$ radio networks will have $r$ instances of this hierarchy of queues to handle the difference in both coverage (kilometers) and link data rate (kbps) from military communication technologies, such as HF (High Frequency), VHF (Very High Frequency) and UHF (Ultra High Frequency). 


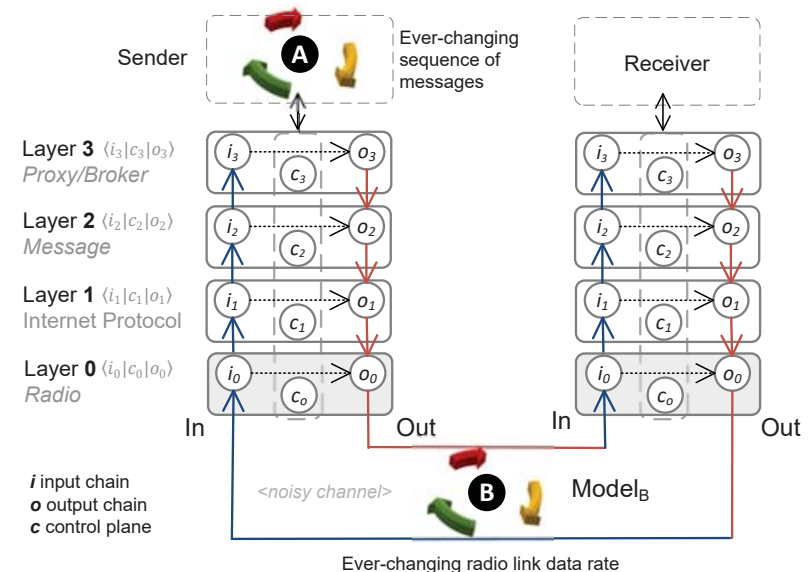

Fig. 1: Ever-changing end-to-end communication scenario

\section{$B$. How to compute the probability of message delivery?}

Let us assume that the network conditions in a communication scenario $C$ is described as the five-tuple $C=$ $\left(\vec{X}_{0}, \mathfrak{B}, \theta, \mathfrak{B}_{T}, \mathfrak{T}\right)$, where $\vec{X}_{0}$ is the initial state vector, $\mathfrak{B}$ is a set of matrices describing $M$ different probability distributions, $\theta$ is the update function, $\mathfrak{B}_{T}$ is a set of transition matrices and $\mathfrak{T}$ is the time distribution of the in-homogeneous Markov model, called Model $_{B}$ as we described in [1].

Now, we are interested in the probability of a message being successfully transmitted from sender to receiver in Fig. 1, while the radio link is changing according to the distribution defined by $C$. For this purpose, our system implements an error correction technique called Reed Solomon Code ( [3], [4]), meaning that if the message consists of $k$ data and $n-k$ redundant packets, the message can be successfully delivered if at least $k$ packets are delivered by sending a selective acknowledgement, which indicates the sequence numbers of the lost packets. If more than $k$ packets are successfully delivered no re-transmission is required. Our goal is to avoid the overhead of re-transmissions by adjusting the system parameters to maximize the probability that at least $k$ packets are transmitted.

Assuming independent errors that might cause packet loss, the probability for the receiver getting any $k$ out of $n$ sent packets is given by the binomial distribution defined as:

$$
\mathbb{P}(X=k)=\left(\begin{array}{l}
n \\
k
\end{array}\right) \cdot p_{0}^{k} \cdot\left(1-p_{0}\right)^{n-k} \quad, 0 \leq k \leq n,
$$

Where $p_{0}$ is the probability that a single packet will be delivered and $X$ is a random variable measuring the number of successfully transmitted packets. The equation holds because we have $\left(\begin{array}{l}n \\ k\end{array}\right)$ possibilities to sample a set of $k$ packets out of $\Omega=\{1, \ldots, n\}$ packets. The probability that the random variable $X$ is in state $k$ is highly dependent on the current link state (link data rate) and thus dependent on the patterns $P_{i}$ sampled from $\operatorname{Model}_{B}$ defined in [1]. As a result, if $E_{1}$ is the event $X \geq k$, meaning that the message can be successfully delivered in the first round and $E_{2}$ is the event that the outer Markov chain of $\operatorname{Model}_{B}$ is in state $s(t)=P_{i}$, we get

$$
P\left(E_{1} \mid E_{2}\right)=P\left(X \geq k \mid s(t)=P_{i}\right)=\frac{P\left(X \geq k \cap s(t)=P_{i}\right)}{P\left(s(t)=P_{i}\right)}
$$

This approach can be extended to compute the probability $p_{2}$ of message delivery (at layer 2 in Fig. 1) during a communication scenario $C$ consisting of $I$ different patterns $P_{1} \cup \cdots \cup P_{i} \cup \cdots \cup P_{I}=C$ by finding an optimum configuration for

$$
p_{2}=P(X \geq k \mid C)=\sum_{i=1}^{I} P\left(X \geq k_{i} \mid P_{i}\right)
$$

in terms of $k_{i}$ and fulfilling the constraint $\sum_{i=1}^{I} k_{i}=k$ at the same time.

\section{THE SOLUTION}

\section{A. Probability as function of packet size within a time window}

Let us sketch a solution for computing the probability of packet delivery $p_{0}\left(T_{w}\right)$ given a time window $T_{w}$ in seconds using the features collected during the communication scenarios generated in [1]. Thus, we assume that the data rate changes of the system follow an in-homogeneous Markov chain represented by Model $_{B}$ with state space $\mathfrak{B}=B_{1}, \ldots, B_{M}$ resulting in a communication scenario $C=\left(\vec{X}_{0}, \mathfrak{B}, \theta, \mathfrak{B}_{T}, \mathfrak{T}\right)$. Again $B_{1}, \ldots, B_{M} \in \mathfrak{B}$ are also Markov chains, each one representing the probability distribution of a specific pattern of data rate changes. Moreover, we assume that the distributions $B_{1}, \ldots, B_{m}$ together with the distribution $\lambda$ are well known and therefore we have access to an oracle knowing the link states $\Sigma=[b(1), \ldots, b(T)]$ at each point in time $t \in \mathfrak{T}=[1, \ldots, T]$.

To describe arbitrary time windows associated to the communication scenario $C$, we define the function $f_{l}(t)=$ $\left[\max \left(0, \sum_{i=1}^{t-1} \tau_{i}-1\right), \sum_{i=1}^{t} \tau_{i}-1\right]$, mapping a point in time $t \in \mathfrak{T}$ to a time interval describing how many seconds the link (inner Markov chain) stays in state $b(t) \in[0, \ldots, 5]$. Supposing that the probability of delivering a packet $p_{0}\left(T_{w}\right)$ is proportional to the amount of bits received within the time window $T_{w}=f_{l}\left(t_{1}\right) \cup f\left(t_{2}\right)$ s.t. $t_{1}, t_{2} \in \mathfrak{T}$ and the packet size is distributed by $\kappa$, the optimal data rate $b_{\text {opt }}(t)$ for almost sure delivery can be calculated using the following equation:

$$
b_{\mathrm{opt}}\left(T_{w}\right)=\frac{\kappa(\mathrm{kb})}{\left|T_{w}\right|(\mathrm{s})}
$$

Using this relation, we can compute the ratio $b_{\text {ratio }}$ between the current data rate $b(t)$ and the optimal data rate $b_{\text {opt }}(t)$ for arbitrary time windows $T_{w}$.

$$
b_{\text {ratio }}=\frac{\sum_{t=t_{1}}^{t_{2}} \frac{f_{l}(t)}{\left|T_{w}\right|} b(t)(\mathrm{kbps})}{b_{\mathrm{opt}}\left(T_{w}\right)(\mathrm{kbps})}
$$

As a result from Eq. (4) and Eq. (5), we can use $b_{\text {ratio }}$ to introduce a function $g\left(t, b(t), b_{\mathrm{opt}}\left(T_{w}\right)\right)$ that computes an initial guess for the probability of packet delivery at layer 0 :

$$
g\left(T_{w}, b(t), b_{\mathrm{opt}}\left(T_{w}\right)\right)=\left\{\begin{array}{l}
0, \quad \text { if } b(t)=0 \quad \forall t \in\left[t_{1}, t_{2}\right] \\
b_{\mathrm{ratio}}, \text { if } \sum_{t=t_{1}}^{t_{2}} \frac{f_{l}(t)}{\left|T_{w}\right|} b(t)<b_{\mathrm{opt}}\left(T_{w}\right) \\
1, \quad \text { if } \sum_{t=t_{1}}^{t_{2}} \frac{f_{l}(t)}{\left|T_{w}\right|} b(t) \geq b_{\mathrm{opt}}\left(T_{w}\right)
\end{array}\right.
$$


This function can be interpreted as follows. If $g=0$, it means that the data rate $b(t)$ is zero for the entire time window $T_{w}$. Obviously no packets can be transmitted and also the probability of packet delivery $p_{0}(t)=0$. If $g=1$, then the capacity of the link in the given time window $T_{w}$ is greater or equals the size $\kappa$ of the packet and as a result $p_{0}(t)>0$. If $g=b_{\text {ratio }}$, then we can solve the equation $\left|T_{w}(t)\right| \cdot \frac{b}{\kappa}=$ $1-g\left(t, b(t), b_{\text {opt }}(t)\right)$ either for fixed $\left|T_{w}(t)\right|$ or $b$ to adjust the system parameters s.t. $g=1$ and as a result $p_{0}(t)>0$. Now, let us assume that $g=1$ and $b(t)>b_{\text {opt }}(t)$ or more precisely $b(t)=(1+\epsilon) \cdot b_{\text {opt }}(t)$. This means that the link is able to handle an additional amount of data $\epsilon \cdot b_{\text {opt }}(t)$. This capacity can be used to increase the probability of packet delivery $p_{0}(t)$ by adding redundancy $r(t)=\epsilon \cdot b_{\mathrm{opt}}(t)$ with $\epsilon=\frac{b(t)-b_{\mathrm{opt}}(t)}{b_{\mathrm{opt}}(t)}$.

From these analysis of $g$, it follows that we can rewrite $p_{0}\left(T_{w}\right)$ as a conditional probability depending on the data rate $b(t)$, the packet size $\kappa$ and the time window $T_{w}$ :

$$
p_{0}\left(T_{w}\right)=\mathbb{P}\left(X=1 \mid b(t), T_{w}, \kappa\right)
$$

To guarantee $p_{0}(t)>0$ even under ever-changing communication scenarios in a laboratory with military hardware, we exploited the properties of function $g$ by varying the parameters of $g$ and as a result also of $p_{0}$ during a set of experiments over stable network conditions. Fig. 2 plots exemplary values of $p_{0}\left(T_{w}\right)$, for two sending rates 0.05 (left) and 2 (right) packets per second, as a function of the nominal data rates $b(t)$ supported by our VHF radios (.6, 1.2, 2.4, 4.8 and $9.6 \mathrm{kbps})$, the packet size $\kappa(1.3 \mathrm{kB})$ and the time window $T_{w}(t)$ (from 0 to $300 \mathrm{sec}$ ). These results over stable conditions are used as ground truth to compute $p_{0}$ and also $p_{2}$ for two communication scenarios including link disconnections in the following sections.
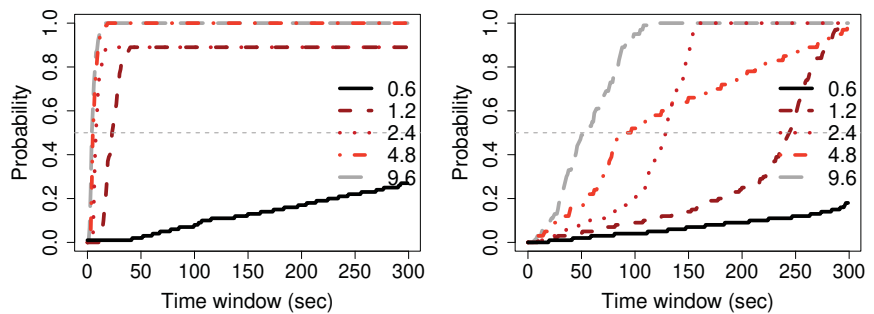

Fig. 2: Probability $p_{0}$ for five nominal data rates using $g$

\section{B. Maximizing the probability of message delivery}

Now, we can use the probability $p_{0}\left(T_{w}\right)$ and the properties of $g$ to maximize the probability of message delivery in everchanging communication scenarios by adding redundancy. For this purpose, we assume that we are given the communication scenario $C$, a message consisting of $k$ data packets and the distribution of the packet size $\kappa$. Furthermore, we consider that we can access the probabilities $p_{0}\left(T_{w}\right)=\mathbb{P}(X=$ $\left.1 \mid b(t), T_{w}(t), \kappa\right)$ for different time windows $T_{w}$ with maximum size $t_{w_{\max }}$ as shown in Fig. 2. Referring to the problem in section II-B, we state an optimization problem to find the maximum value of $p_{2}$ given $C$ :
Problem III.1 (Maximizing the probability of message delivery with a minimum amount of redundancy).

$$
\begin{aligned}
& \min _{r} \max _{\mathbb{P}} \mathbb{P}(X \geq k \wedge Y=n-k \mid C) \\
= & \min _{r} \max _{\mathbb{P}} \sum_{i=1}^{I} \mathbb{P}\left(X=k_{i} \wedge Y=n_{i}-k_{i} \mid P_{i}\right) \\
& \text { subject to: } \\
& \sum_{i=1}^{I} k_{i} \geq k, \sum_{i=1}^{I} n_{i}-k_{i}=r, n_{i}-k_{i} \geq 0, n_{i}, k_{i} \geq 0
\end{aligned}
$$

Here, the patterns $P_{i} \subseteq \Sigma$ of link data rates are generated by the matrices $B_{m} \in \mathfrak{B}$ defining the communication scenario $C$. The parameters $k_{i}$ represent the number of packets that should be transmitted, assuming that the link states change according to pattern $P_{i}$. Since we are interested in sending a message that consists of $k$ packets, the parameters $k_{i}$ must sum up to $k$. The idea is to search for the best strategy to send $k$ data packets during a scenario composed of different patterns $P_{i}$ by using an optimal amount of redundancy characterised by the random variable $Y=n_{i}-k_{i}$, meaning that $Y$ maximizes $\mathbb{P}\left(X=k_{i} \wedge\right.$ $\left.Y=n_{i}-k_{i} \mid P_{i}\right)$ by fulfilling the constraint $\sum_{i=1}^{m} k_{i} \geq k$ and minimizing the overall amount of redundancy $r=\sum_{i=1}^{m} n_{i}-$ $k_{i}$ at the same time. The following theorem shows that for any arbitrary communication scenario generated by Model $_{B}$, there exists an algorithm s.t. we can find an optimum solution for the problem defined by Eq. (8).

Theorem III.1.1 (Optimized message delivery in ever-changing communication scenarios). Given a non-empty, ever-changing communication scenario $C=\left(\vec{X}_{0}, \mathfrak{B}, \theta, \mathfrak{B}_{T}, \mathfrak{T}\right)$, the probabilities $\mathbf{P}_{0}$ for different end-to-end delays $1, \ldots, t_{w_{\max }}$ over stable system conditions, the number $k>0$ of data packets defining the length of a message Msg, the distribution of the the packet size $\kappa$ and the maximum end-to-end delay $t_{w_{\max }}, \mathrm{MAXPROB}$ (Alg. 1) computes an optimum solution to the problem defined by Eq. (8).

Proof. Let $C, k, \kappa$ and $t_{w_{\max }}$ be as defined in the statement of the theorem. First we show that Alg. 1 finds the maximum probability for delivering at least $k$ packets by using a minimum amount of redundancy or in other words Alg. 1 finds an optimum solution to problem Eq. (8). The idea is to proof inductively that for each possible end-to-end delay $1 \leq t_{w} \leq t_{w_{\max }}, p_{\text {best }}$ and $r_{\text {best }}$ are always optimal. Initially, we sample the sequence of states $\Sigma$ consisting of patterns $P_{i}, i \in I$ using the Sample algorithm from [1]. For the correctness of this algorithm we refer to our previous investigation. Moreover, the data structures $S_{\mathrm{pt}}, S_{\mathrm{k}}$ storing the probabilities for each pattern $P_{i}$ and packet size $k$ are empty and the variable $k$ is set to the number of data packets of the Msg (lines 2-5).

Now, let $t_{w}=1$, then lines 7-12 guarantee that we compute the maximum amount of redundancy $r_{i}$ that the system can handle and the corresponding probability $\mathbb{P}\left(X \geq k_{i} \wedge Y=n_{i}-\right.$ $\left.k_{i} \mid P_{i}\right)$ with $n_{i}-k_{i}=\left\lfloor\frac{r_{i}}{\kappa}\right\rfloor$ by using the probabilities $p_{0} \in \mathbf{P}_{0}$ for each possible combination of a pattern $P_{i}$ and number of packets $k_{i} \leq k$. The probabilities for fixed pattern $P_{i}$ are stored 
in the data structure $S_{k}$ and added to $S_{p t}$ afterwards. As a result, after line 13 of the algorithm, $S_{p t}$ stores all probabilities for delivering $k$ packets by considering each possible pattern $P_{i} \subseteq \Sigma$. In sequence (line 15), the algorithm computes all possible configurations maximizing $\sum_{i=1}^{I}\left(\mathbb{P}\left(X \geq k_{i} \wedge Y=\right.\right.$ $\left.\left.n_{i}-k_{i} \mid P_{i}\right)\right)$, s.t. $\sum_{i=1}^{I} k_{i} \geq k$ and each pattern $P_{i}$ is considered only once. We keep the configuration that uses a minimum amount of redundancy $r_{\min }=\sum_{i=1}^{I} r_{i}=n_{i}-k_{i}$. Since $p_{\max }>p_{\text {best }}$ is obviously true, we update $p_{\text {best }}$ and $r_{\text {best }}$. By line $15\left(p_{\text {best }}, r_{\text {best }}\right)$ is an optimal configuration to to problem Eq. (8) for $t_{w}=1$.

The induction step $\left(t_{w}\right) \rightarrow\left(t_{w}+1\right)$ can be done along the same lines. Since we are updating $\left(p_{\text {best }}, r_{\text {best }}\right)$ only if the algorithm finds a higher probability $p_{\max }$, the tuple $\left(p_{\text {best }}, r_{\text {best }}\right)$ is always optimal. As a result Alg. 1 is correct and outputs the maximum probability for delivering at least $k$ packets by adding a minimized amount of redundancy that does not exceed the capacity of the system. It remains to show that the algorithm terminates for each possible configuration of $C, k, \kappa$ and $t_{w_{\max }}$. But since $C$ is always finite and $k, \kappa$ and $t_{w_{\max }}$ this is trivial.

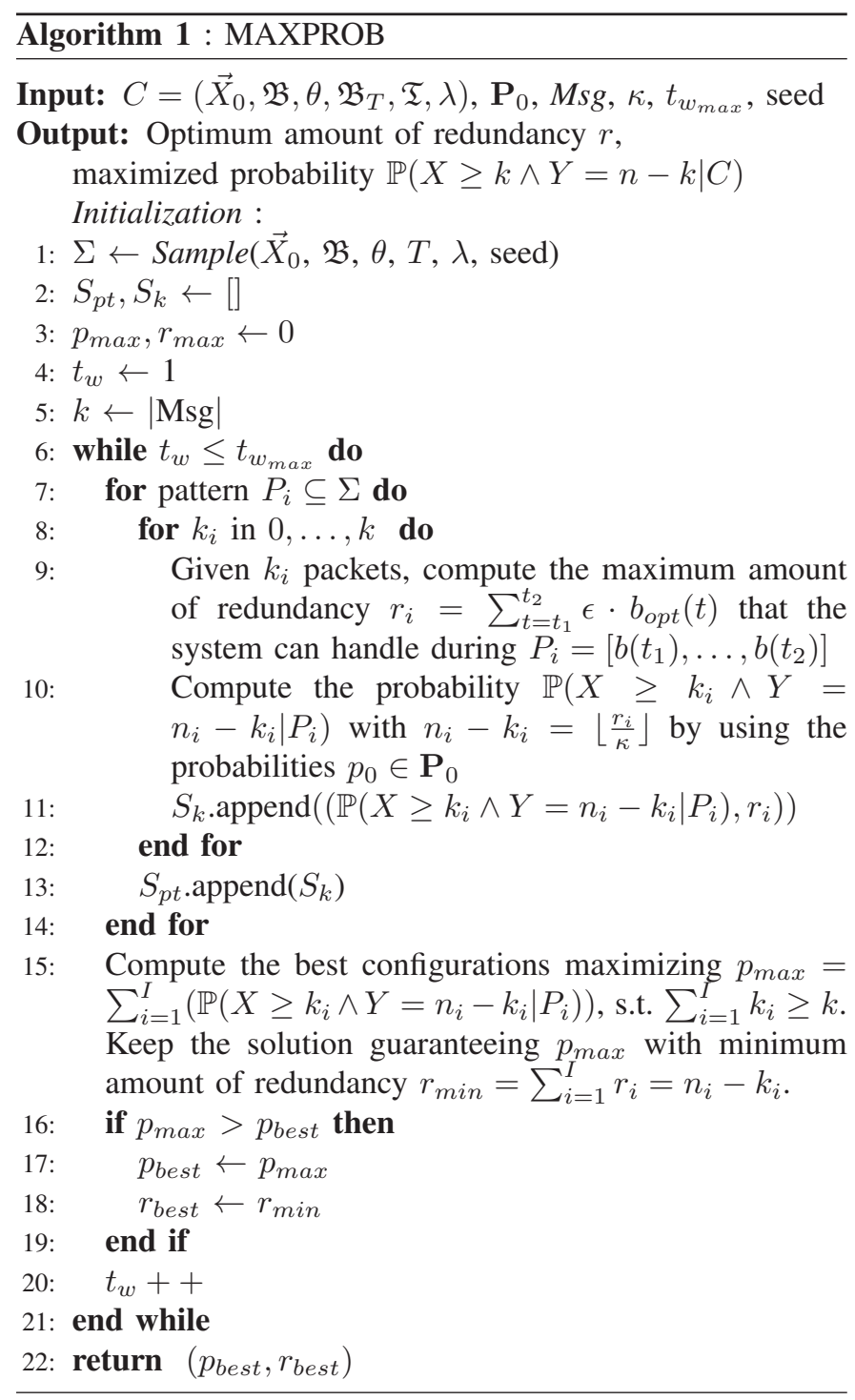

\section{Multi-layer stochastic uncertainty model}

Given the probabilities $p_{i}\left(T_{w}\right) i \in$ layers $[0,2]$ and the distribution of the packet size $\kappa$, finally we introduce a two layered stochastic uncertainty model over time $\mathfrak{T}$. Referring to [5], we are modeling the tactical system uncertainty and, since the probability $p_{i}\left(T_{w}\right)$ depends on the data rates $b(t)$ generated by $\operatorname{Model}_{B}$, the stochastic uncertainty model can be defined in a similar way. To this end, we assume that $\mathfrak{M}$ is an instance of Model $_{B}$ and that we are given the 5-tuple $C=\left(\vec{X}_{0}, \mathfrak{B}, \theta, \mathfrak{B}_{T}, \mathfrak{T}\right)$ required to sample the sequence $\Sigma$ by computing $\phi_{\text {sample }}$ as proposed in [1].

Now we can define two functions to convert the instance $\mathfrak{M}$ to an instance $\mathfrak{U}$ of an stochastic model describing the uncertainty of the underlying tactical system by replacing the system states $s_{\text {in }}(t) \in[0, \ldots, 5]$ (radio link data rates) of the inner Markov chain of $\mathfrak{M}$ by probabilities $p_{0}$ and the pattern $P_{j}$ representing the states of the outer Markov chain by $p_{2}$. To this end, we define the function $\psi_{i n}$ mapping each system state $s_{\text {in }}(t)$ to a probability $p_{0}$ as:

$$
\begin{aligned}
\psi_{\text {in }}:\left(s_{\text {in }}(t), t\right) \rightarrow & \\
& \left(\mathbb{P}\left(X=1 \mid b(t)=s_{\text {in }}(t), T_{w}=f_{l}(t), \kappa\right) .\right.
\end{aligned}
$$

Moreover, we can fix the state of the outer Markov chain for a time interval $\left[t_{1}, t_{2}\right]$ with $0 \leq t_{1}<t_{2} \leq$ $T, t_{1}, t_{2} \in \mathfrak{T}$, meaning that the system follows a pattern $P_{j}=\left[s_{\text {in }}\left(t_{1}\right), \ldots, s_{\text {in }}\left(t_{2}\right)\right]$ generated by a single matrix $B_{j} \in$ $\mathcal{B}$. Given the size $k$ of a message, we can now replace $P_{j}$ by the maximized probability $p_{2}$ using $\psi_{\text {out }}$ defined as:

$$
\begin{aligned}
& \psi_{\text {out }}:\left(P_{j}, t_{1}, t_{2}, k\right) \rightarrow \\
& \mathbb{P}\left(X \geq k \mid P_{i}=P_{j}, T_{w}=f_{l}\left(t_{1}\right) \cup f_{l}\left(t_{2}\right), \kappa\right)
\end{aligned}
$$

As a result, this model describes the distribution of uncertainty for packet delivery in the lower layer 0 and the probability of message delivery in the higher layer 2 .

\section{RESUlts}

In this section, we discuss experimental results observed in the end-to-end communication scenarios earlier shown in Fig. 1. We reused two loop patterns, $L_{1}$ and $L_{2}$, and the VHF network from the experimental setup described in [1]. Both patterns of change include very low link data rates (i.e 0.6 and $1.2 \mathrm{kbps}$ ) and even link disconnections (0 kbps) resulting in packet loss. Here, we demonstrate how redundancy can improve the message/packet delivery over the loop patterns $L_{1}$ (5 \% loss) and $L_{2}$ (54\% loss), both with 200 states updated every 10 seconds. We have chosen these two loop patterns because they concentrate the link disconnections in the middle of the experiment. The messages were composed by 1, 3, 9, 18 and 54 packets. The packet rate, at the sender radio, was distributed according to $(0.05,0.075,0.1,0.2,0.5,1,2)$ packet/second.

The goal is to illustrate the numerical output of our model discussed earlier in Section III. Table I lists the results for 0.2 packet/second, and four levels of redundancy 0,100 , 
TABLE I: Probability of message delivery (0.2 packetssecond)

\begin{tabular}{lc|ccccc}
\hline \multirow{2}{*}{} & \multirow{2}{*}{$(\boldsymbol{t})$} & & \multicolumn{5}{c}{ Number of packets per message } \\
& & 1 & 3 & 9 & 18 & 54 \\
\hline$L_{1}^{*}$ & \multirow{2}{*}{$0 \%$} & $.69( \pm .11)$ & $.33_{( \pm .08)}$ & $.04( \pm .01)$ & $.00( \pm .00)$ & $.00( \pm .00)$ \\
$L_{2}^{*}$ & & $.63( \pm .09)$ & $.25( \pm .05)$ & $.02( \pm .00)$ & $.00( \pm .00)$ & $.00( \pm .00)$ \\
\hline$L_{1}$ & \multirow{2}{*}{$0 \%$} & $.49( \pm .11)$ & $.30( \pm .08)$ & $.04( \pm .01)$ & $.00( \pm .00)$ & $.00( \pm .00)$ \\
$L_{2}$ & & $.61( \pm .09)$ & $.25( \pm .05)$ & $.02( \pm .00)$ & $.00( \pm .00)$ & $.00( \pm .00)$ \\
\hline$L_{1}$ & \multirow{2}{*}{$100 \%$} & $.69( \pm .12)$ & $.90( \pm .17)$ & $.97( \pm .21)$ & $.99( \pm .24)$ & $.99( \pm .26)$ \\
$L_{2}$ & & $.71_{( \pm .11)}$ & $.86_{( \pm .14)}$ & $.92_{( \pm .18)}$ & $.96( \pm .20)$ & $.99( \pm .22)$ \\
\hline$L_{1}$ & \multirow{2}{*}{$200 \%$} & $.82( \pm .12)$ & $.99( \pm .14)$ & $.99( \pm .16)$ & $.99( \pm .16)$ & $.99( \pm .16)$ \\
$L_{2}$ & & $.76( \pm .11)$ & $.98_{( \pm .14)}$ & $.99( \pm .15)$ & $.99( \pm .15)$ & $.99( \pm .16)$ \\
\hline$L_{1}$ & \multirow{2}{*}{$500 \%$} & $.96( \pm .10)$ & $.99( \pm .12)$ & $1.0( \pm .12)$ & $1.0( \pm .13)$ & $1.0( \pm .13)$ \\
$L_{2}$ & & $.97( \pm .10)$ & $.99( \pm .11)$ & $1.0( \pm .12)$ & $1.0( \pm .12)$ & $1.0( \pm .13)$ \\
\hline
\end{tabular}

200 and $500 \%$. In this table, $L_{1}^{*}$ and $L_{2}^{*}$ are the baseline results computed supposing that $p_{0}$ is known for these patterns of change. In contrast, all other results for $L_{1}$ and $L_{2}$ are computed using $p_{0}$ from the nominal data rates supported by our VHF radios, as plotted earlier in Fig. 2. Using the results from these experiments, we computed the probability of message delivery for different maximum end-to-end delay $\left|T_{w}\right| \in\{1, \ldots, 300\}$ using Alg. 1 .

Notice that without redundancy, $r(t)=0 \%$, the probability of message delivery is about $60 \%$ for messages with 1 packet and goes decreasing until $0 \%$ for messages with 18 or more packets. This fact motivated the present investigation and is visible in the first four lines in Table I, listing the probabilities measured $\left(L_{1}^{*}\right.$ and $\left.L_{2}^{*}\right)$ and the probabilities computed using Alg. 1 (lines 3 and 4 in the table). Moreover, we observed that adding redundancy can significantly increase the probability of message delivery for both loop scenarios. For messages consisting of a single packet, we need up to $500 \%$ of redundancy to guarantee reliable message delivery. For messages consisting of 3 or more packets, we see that even $100 \%$ redundancy can lead to satisfying results (i.e. probabilities close to 1 ).

Fig. 3 complements the results from this table by showing the probability for message delivery as a function of different time windows $T_{w}$ for six messages sizes, namely $1,3,9$, 18 and 54 packets per message. In this figure, there are two examples representing $100 \%$ (left) and $200 \%$ (right) for $L_{1}$ showing that the probabilities converge with respect to the size of the maximum end-to-end delay $\left|T_{w}\right|$; also called time window. This is an important result, because it yields to the assumption that we can replace the parameter $t_{w_{\max }}$ by another parameter describing the conversion rate of Alg. 1 .
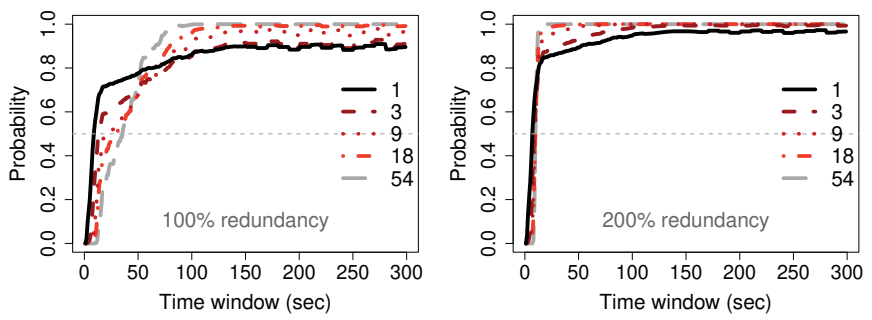

Fig. 3: Probability as function of time window

\section{CONCLUSION}

This letter introduced a stochastic model to maximize the probability of message/packet delivery using the hierarchy of layers from modern tactical systems. The goal was to estimate the optimum redundancy level to mitigate packet loss in communication scenarios with link disconnection therefore increasing the probability of delivering messages. Thus, we started with the hypothesis that transport protocols can use our model to proactively add redundancy to reduce the packet loss observed during radio link disconnections. Our hypothesis was verified with experiments sending messages with different sizes through a VHF link with data rate changing in two different patterns. The experimental results suggest that our stochastic model can compute close to optimal parameters for a transport protocol using redundancy to overcome packet loss during link disconnections, also avoiding data overhead from packet acknowledgements and packet re-transmissions.

\section{REFERENCES}

[1] R. R. F. Lopes, J. F. Loevenich, P. H. Rettore, S. M. Eswarappa, and P. Sevenich, "Quantizing radio link data rates to create ever-changing network conditions in tactical networks," IEEE Access, vol. 8, pp. 188015-188 035, September 2020.

[2] R. R. F. Lopes, P. H. Balaraju, P. H. Rettore, and P. Sevenich, "Queuing over ever-changing communication scenarios in tactical networks," IEEE Transactions on Mobile Computing, pp. 1-15, June 2020, (early access).

[3] N. Tang and Y. Lin, "Fast encoding and decoding algorithms for arbitrary $(n, k)$ Reed-Solomon codes over $\mathbb{F}_{2} m$, , IEEE Communications Letters, vol. 24, no. 4, pp. 716-719, 2020.

[4] Y. L. Grushevsky and G. F. Elmasry, "Adaptive RS codes for message delivery over an encrypted mobile network," IET Communications, vol. 3, no. 6, pp. 1041-1049, 2009.

[5] C. E. Shannon, "A mathematical theory of communication," The Bell System Technical Journal, vol. 27, no. 3, pp. 379-423, 1948. 